\title{
A fast cold-start method of GPS receiver based on satellite orbit prediction
}

\author{
Ruihong Yang ${ }^{1}$, Zhongguo Song ${ }^{1}$, Jinsheng Zhang ${ }^{2}$ and Xiaoli Xi ${ }^{1}$ \\ ${ }^{1}$ Xi'an University of Technology, Xi'an, China \\ ${ }^{2}$ Xi'an research inst of hi-tech, Xi'an, China
}

\begin{abstract}
Time to first fix (TTFF) is one of the crucial indicators to evaluate the performance of a GPS receiver. In this paper, an orbit prediction algorithm to reduce the TTFF of GPS receivers without a network connection is presented. Satellite orbit is predicted by numerically integrating the satellite's equation of motion. Satellite's initial position and velocity value, as well as the Earth's polar motion parameters, used in prediction correspond to the locally collected previous broadcast ephemeris. As the solar radiation pressure (SRP) is one of the most critical factors causing orbit prediction error, an empirical SRP model with two parameters that change with the movement of satellite and Earth is also described. The presented algorithm is verified by GPS satellites using the initial conditions divided from three groups of broadcast ephemeris data. Simulation results show that, with the SRP model described in this paper the satellite's position error limited to 30 meters within 7 days prediction.
\end{abstract}

\section{Introduction}

Start navigation immediately is expected for people once their GPS receivers are turned on. However, it typically needs about 30 seconds for the receiver to provide the first position information even in an ideal receiving environment, during to the three first sub-frames which contain ephemeris data takes 18 seconds to transmit and the satellite sends it once every 30 seconds. If the signal is blocked by trees or buildings during the ephemeris collection and the TTFF can even take up to several minutes, which is unacceptable especially in an emergency or a special situation $[1,2]$.

Due to the time needed for the reception of the ephemeris data is the main reason for long TTFF, one method to reduce it is to provide satellite position information by orbit prediction. One technique of satellite orbit prediction that runs entirely on the receiver without any external assistance has been implemented in commercial products [3]. Reference [4] and [5] present a fully detailed description of an autonomous satellite orbit prediction method which only needs a few locally collected previous broadcast ephemeris data as a starting condition. This paper aims to develop a related method and make its efficiency and precision are competitive when compared to others.

In this paper, a practical orbit prediction algorithm to reduce the TTFF of a GPS receiver is presented. As the SRP is one of the most critical factors causing orbit prediction error, an enhanced sphere model that used for calculating SRP is adopted to improve the accuracy of orbit prediction. Also, to minimize the computational burden of GPS receiver, a gravity acceleration approximation function (GAAF) to calculate the Earth's gravitational acceleration is utilized to substitute the traditional spherical harmonic representation of the gravity field [6]. Simulation results show that the orbit prediction algorithm proposed in this paper can provide a trusted satellite position and significantly reduce the TTFF of a GPS receiver.

\section{Force models}

A Large number of different forces in the equation of motion will lead to a computationally heavier algorithm, which is not desired in a conventional GPS receiver. Therefore, only the four forces (including the Earth's gravitation, solar gravitation, lunar gravitation, and solar radiation pressure) that primarily affect the GPS satellite are taken into account.

The satellite's equation of motion used in this work is expressed as

$$
\mathbf{a}_{\mathrm{Sat}}=\mathbf{a}_{\mathrm{Earth}}+\mathbf{a}_{\mathrm{Sun}}+\mathbf{a}_{\mathrm{Moon}}+\mathbf{a}_{\mathrm{SRP}}
$$

where $\mathbf{a}_{\text {Sat }}$ denotes the acceleration of the satellite, $\mathbf{a}_{\text {Earth }}$ is the acceleration due to the Earth's gravitation, $\mathbf{a}_{\text {Sun }}$ and $\mathbf{a}_{\text {Moon }}$ is the acceleration due to solar gravitation and lunar gravitation respectively. The term $\mathbf{a}_{\mathrm{SRP}}$ is the acceleration caused by solar radiation pressure.

Once the satellite position and velocity at epoch $t_{0}$ is known for a satellite, one can predict the satellite orbit at an epoch $t_{k}$ by integrating (1) with respect to time. 


\subsection{Earth's gravitation}

For the Earth gravity, we utilized the GAAF method to compute the gravitational acceleration replacing the traditional spherical harmonic representation of the gravity field. The GAFF introduced an equation to represent gravity accelerations in terms of instant twobody acceleration for an instant pseudo-center for the earth. Pseudo-center location is represented by polynomials which as a function of height at fixed latitude and longitude. Pseudo-center at any point of interest can be calculated by classical six-point bivariate interpolation in latitude and longitude at a fixed height. Thus the calculation of gravitational acceleration is simplified to calculate the two-body acceleration. GAAF can significantly improve the computational efficiency in orbit prediction, more details about it can be found in [7]. In this work, we used the Earth gravitational model 2008 (EGM2008) geopotential up to degree and order 70 to generate the polynomial coefficient of pseudo-center calculation [8].

\subsection{Solar and lunar's gravitation}

Consider the Moon and the Sun as point masses. The acceleration due to a celestial body's gravitation is expressed as

$$
\mathbf{a}_{\mathrm{Cb}}=G M_{\mathrm{Cb}}\left(\frac{\mathbf{r}_{\mathrm{Cb}}-\mathbf{r}_{\mathrm{Sat}}}{\left|\mathbf{r}_{\mathrm{Cb}}-\mathbf{r}_{\mathrm{Sat}}\right|^{3}}-\frac{\mathbf{r}_{\mathrm{Cb}}}{\left|\mathbf{r}_{\mathrm{Cb}}\right|^{3}}\right)
$$

where $G$ is the gravitational constant, $M_{C b}$ and $\mathbf{r}_{\mathrm{Cb}}$ is the mass and position of the celestial body, $\mathbf{r}_{\mathrm{Sat}}$ is the position of the satellite.

\subsection{Solar radiation pressure}

The traditional sphere model of the SRP can be expressed as

$$
\mathbf{a}_{\mathrm{SRP}}=-v \frac{C}{\left|\mathbf{r}_{\mathrm{s}}\right|^{2}}(1+\eta) \mathbf{e}_{\mathrm{D}}
$$

where the factor $v$ is the shadow function based on the conical shadow model described in [9]. Vector $\mathbf{r}_{\mathrm{s}}$ points from the satellite to the Sun and $\mathbf{e}_{\mathrm{D}}$ is a unit vector from the satellite to the Sun. $\eta$ is the reflectivity coefficient of the satellite. The constant $C$ is

$$
C=P_{\text {Sun }} \cdot \frac{A}{m} \cdot A U^{2}
$$

where $A U$ is the astronomical unit, $P_{\text {Sun }}$ is the solar radiation pressure at the distance of $1 A U$ from the Sun, $A$ is the surface area of the satellite and $m$ is the satellite's mass.

The simple sphere model described in (3) is widely used for calculating the SRP. However, it's accuracy need to be improved to meet the application with high precision requirements.

Taking the y-bias acceleration into consideration and then the SRP model we used in this paper can be written as

$$
\mathbf{a}_{\mathrm{SRP}}=v\left(-\frac{C}{\left|\mathbf{r}_{\mathrm{s}}\right|^{2}}\left(1+\alpha_{1}\right) \mathbf{e}_{\mathrm{D}}+\alpha_{2} \mathbf{e}_{\mathrm{Y}}\right)
$$

where $\mathbf{e}_{\mathrm{Y}}$ is the direction vector along the satellite's solar panel axis, and it can be obtained by

$$
\mathbf{e}_{\mathrm{Y}}=\frac{\mathbf{r}_{\mathrm{Sat}} \times \mathbf{r}_{\mathrm{s}}}{\left|\mathbf{r}_{\mathrm{Sat}} \times \mathbf{r}_{\mathrm{s}}\right|}
$$

$\alpha_{1}$ and $\alpha_{2}$ are SRP model parameters which need to be estimated. Although these two parameters change very little and are usually used as constants in a few days orbit prediction, in fact they are varying with the movement of satellite and Earth. Neglecting this variation will cause some errors.

The variation of SRP model parameters with the angle of Sun-satellite-Earth can be expressed by the following equation [10]:

$$
\left\{\begin{array}{c}
\alpha_{1}=a+b \varepsilon^{2}+c \varepsilon^{4}+d \varepsilon^{6} \\
\alpha_{2}=e+f \varepsilon^{2}+g \varepsilon^{4}
\end{array}\right.
$$

where $\varepsilon$ is the angle of the Sun-satellite-Earth, $a \square g$ is the polynomial coefficients.

\section{Procedures for orbit prediction}

\subsection{Estimation of SRP model parameters}

Large amounts of data and computation power are needed in the estimation of the SRP parameters. Thus we have to estimate the SRP parameters offline on a server.

The parameters $\alpha_{1}$ and $\alpha_{2}$ in (5) are satellite-specific that need to be estimated separately for each satellite. Precise ephemeris from GPS weeks 1895-1925 is used as measurement data for this estimation [11]. For one satellite, a time series of parameter estimates are obtained by taking separate consecutive 1-hour arcs and estimating the parameters for each arc using an iterative leastsquares minimization algorithm or the Extended Kalman Filter. For each arc the output of the estimator is taken as the estimated value. Then use (7) to fit the polynomial coefficients to all estimated values. By doing this processing for each satellite, the polynomial coefficients for calculating the SRP for all satellites are obtained.

In the estimation of SRP model parameters, the initial position and velocity of the satellite are taken from precise ephemeris, and the Earth Orientation Parameters (EOP) needed in coordinate system transformation are obtained from the homepage of International Earth Rotation and Reference Systems Service (IERS) [12].

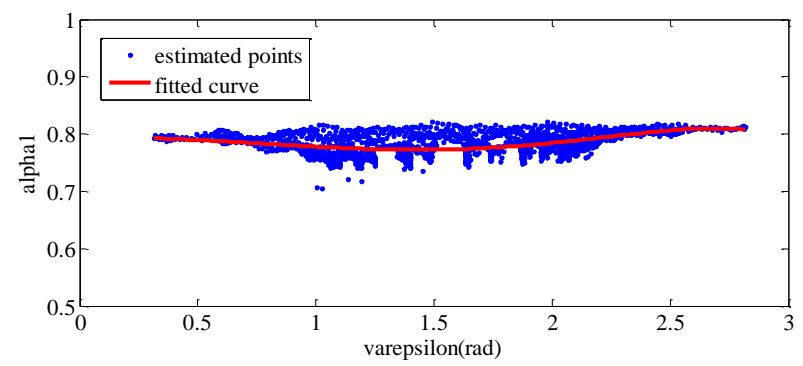

Figure 1. SRP parameters $\alpha_{1}$ of GPS PRN2 


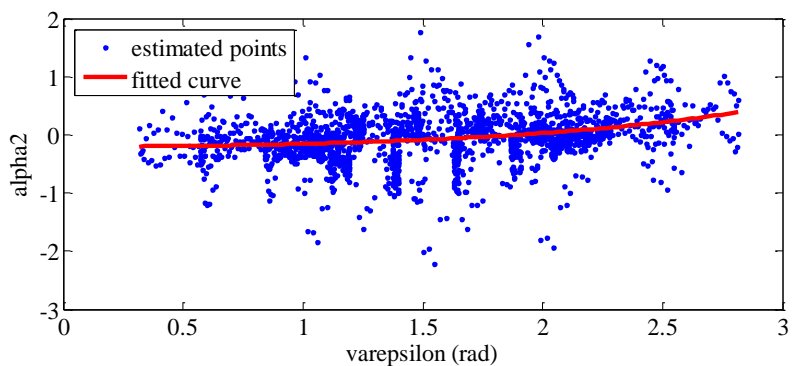

Figure 2. SRP parameters $\alpha_{2}$ of GPS PRN2

Fig. 1 and 2 show the variation of $\alpha_{1}$ and $\alpha_{2}$ with $\varepsilon$ angle for GPS PRN2.

\subsection{Initialization}

For orbit prediction, the forces model and the initial position and velocity significantly affect the prediction accuracy. Models and algorithms for calculating the forces have been discussed in the previous sections.

The initial position and velocity of satellite play a key role in orbit prediction. However, the position and velocity of satellite calculated by broadcast ephemeris are more inaccurate, and it cannot be used as an initial state directly. For this reason, the least squares algorithm is used to get an estimation of the initial state. In addition, the EOP used to calculate the matrix of coordinate system transformation in orbit prediction is unknown. So it has to be estimated together with the initial state from the broadcast ephemeris.

To minimize the number of parameters to be estimated, and improve the accuracy of the least squares solution, we only make a simple correction by antenna offsets for the initial position calculated by broadcast ephemeris. Furthermore, the time difference between Universal Time UT1 and Coordinated Universal Time is always set to zero. Thus only the initial velocity of satellite and polar motion parameters need to be estimated.

In this paper, we solve the unknown polar motion parameters and the initial velocity by fitting our model to multiple positions calculated by broadcast ephemeris. The variation of polar motion parameters in a few days orbit prediction is ignored.

For one satellite, the vector of unknowns is

$$
\mathbf{x}=\left[\begin{array}{c}
\mathbf{v}_{0} \\
x_{p}\left(t_{0}\right) \\
y_{p}\left(t_{0}\right)
\end{array}\right]
$$

where $\mathbf{v}_{0}$ is the initial velocity of the satellite, $x_{p}\left(t_{0}\right)$ and $y_{p}\left(t_{0}\right)$ are the polar motion parameters at time instant $t_{0}$. As measurements we have the satellite's positions $\mathbf{y}_{k}$ at time instants $t_{1}, t_{2}, \ldots, t_{m}$ which can be described by equation

$$
\mathbf{y}_{k}=\mathbf{h}_{k}(\mathbf{x})+\boldsymbol{\varepsilon}_{k}, \quad k=1,2, \ldots, m .
$$

Vectors $\boldsymbol{\varepsilon}_{k}$ are the differences between the measured position at instant $t_{k}$ and the position that was predicted using the function defined by equation

$$
\mathbf{r}\left(t_{k}\right)=\mathbf{h}_{k}(\mathbf{x})=\mathbf{f}\left(t_{0}, t_{k}, \mathbf{r}_{0}, \mathbf{v}_{0}, x_{p}\left(t_{0}\right), y_{p}\left(t_{0}\right)\right)
$$

where $\mathbf{f}$ is the function that predicts satellite states by integrating satellite's equation of motion and carrying out the required transformations between the Earth-fixed, Earth-centered reference frame and the inertial reference frame. Then we need to solve the nonlinear least squares fitting problem

$$
\hat{\mathbf{x}}=\arg \min _{x}\left(\sum_{k} \boldsymbol{\varepsilon}_{k}^{T}(\mathbf{x}) \cdot \boldsymbol{\varepsilon}_{k}(\mathbf{x})\right)
$$

\subsection{Orbit prediction}

After getting the estimated initial state and the earth orientation parameters, satellite position and velocity at any time can be calculated by integrating numerically. Various numerical integration methods have been used to solve the satellite's equation of motion. In this paper, the $8^{\text {th }}$ order Runge-Kutta and $12^{\text {th }}$ order Adams-Cowell methods are adopted to solve the satellite's equation of motion [13], because they are generally regarded as one of the most effective methods in satellite orbit prediction. These predicted orbit values can be used directly or fitted to broadcast ephemeris provided to the receiver.

\section{Simulation result}

The position errors for 7 days orbit prediction for GPS PRN 1 are shown in Fig. 3. The red line is the result with the SRP model which parameters varying with $\varepsilon$ angle as described in (7). And the black line is the 

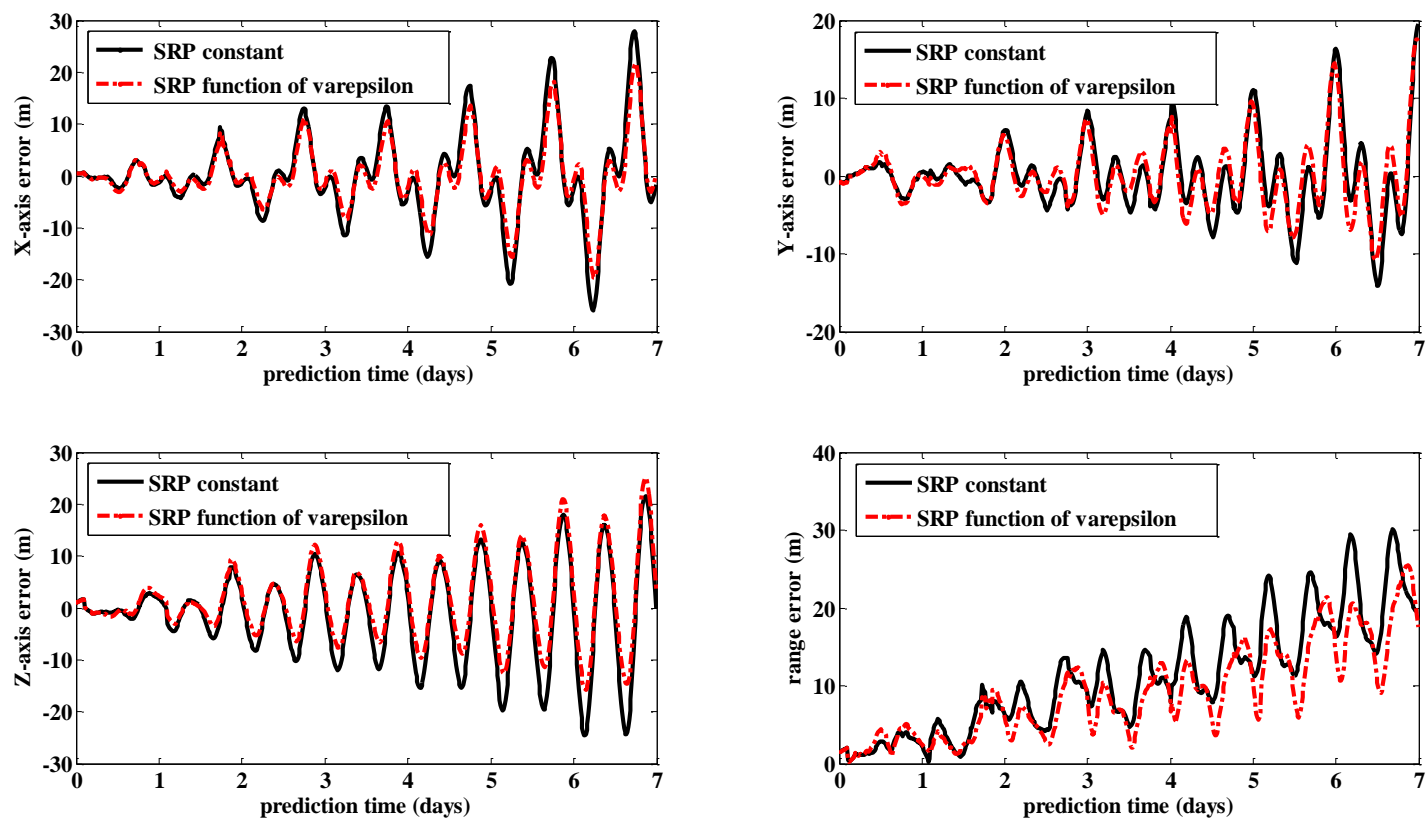

Figure 3. 7-days orbit prediction for GPS PRN1.

result of same orbit prediction, but using the constant parameter SRP model introduced in [5]. The initial points of prediction are calculated by each broadcast from 08:00:00 to 12:00:00 (GPS time) on 13 Jan 2017. The orbit information of initial points are improved by an least squares algorithm, then predict for 7 days with our propagator, and finally compare the predicted orbit with the precise orbit product to obtain the orbit prediction error.

Fig. 3 shows that with the SRP parameters varying as $\varepsilon$ angle change, orbit prediction result became better than using constant SRP parameters, and the peak values of error are smaller.

The orbit prediction results show in Fig. 3 are summarized in Table 1.

Table 1. 7-days orbit prediction error for GPS PRN1.

\begin{tabular}{cccc}
\hline SRP Model & $\begin{array}{c}\text { Average } \\
\text { error(m) }\end{array}$ & $\begin{array}{c}\text { Variance } \\
\text { of } \\
\text { error }\left(\mathbf{m}^{2}\right)\end{array}$ & $\begin{array}{l}\text { Maximum } \\
\text { error(m) }\end{array}$ \\
\hline $\begin{array}{c}\text { SRP constant } \\
\text { SRP function } \\
\text { of } \varepsilon\end{array}$ & 11.74 & 64.44 & 37.00 \\
\hline
\end{tabular}

\section{Conclusion}

The TTFF of receivers can be significantly reduced if the satellite's position and velocity information can be obtained by some alternative ways instead of reading the navigation message send by the satellite. This paper presents an orbit prediction algorithm which can run on receivers whose network connections are nonexistent.

Satellite orbit can be predicted by numerically integrating the satellite's equation of motion. In this work, only the four forces (including the Earth's gravitation, solar gravitation, lunar gravitation, and solar radiation pressure) that primarily affect the GPS satellite are taken into account. Satellite's initial position and velocity value, as well as the Earth's polar motion parameters, used in prediction correspond to the locally collected previous broadcast ephemeris. In this paper, an empirical SRP model with two parameters that change with the movement of satellite and Earth is presented to improve the accuracy of the orbit prediction. The procedure of estimating the SRP parameters, and its curve fitting were described in detail.

The orbit prediction algorithm is verified by GPS satellites using the initial position and velocity as well as polar motion parameters divided from three groups of the broadcast ephemeris data. As an example, the results of orbit prediction for GPS PRN1 with the SRP model described in this paper were show. From it we can see that the satellite's position error limited to 30 meters within 7 days prediction. What's more, with the SRP parameters varying with $\varepsilon$ angle, orbit prediction result became better than using constant SRP parameters, and the peak values of error are smaller.

\section{Acknowledgment}

This work was supported in part by the National Natural Science Foundation of China (51707152), in part by the China Postdoctoral Science Foundation funded project (2014M562522XB), in part by the Shaanxi Province Natural Science Foundation of China (2016JQ4023), in part by the Scientific Research Program Funded by Shaanxi Provincial Education Department (15JK1523), and in part by the Scientific Research Project Foundation of Xi'an University of Technology (2016TS024). 


\section{References}

1. Y. Li, Z. Nie, S. Chen, Y. Gao, Proceedings of the ION 2015 Pacific PNT Meeting, 117-130(2015)

2. M. Lytvyn, A. Kemetinger and P. Berglez, Satellite Navigation Technologies \& European Workshop on GNSS Signals and Signal Processing, 1-6(2012)

3. P.G. Mattos, ION GNSS $21^{\text {st }}$. International Technical Meeting of the Satellite Division, 204-211(2008)

4. H. Lu, J. Zhou, C. Yan and A. Peng, 2017 11th IEEE International Conference on Anti-counterfeiting, Security, and Identification (ASID), 150-153(2017)

5. J. Ala-Luhtala, M. Seppänen, R. Piché, Position Location and Navigation Symposium, 568-575(2012)

6. Wu Z G, Yang J Z, Peng H J, Sci Sin Tech, 12321241(2016)

7. R. S. Hujsak, Adv. Astronaut. Sci,93,335-349(1996)
8. NGA EGM2008 model coefficients, Available online: http://earthinfo.nga.mil/GandG/wgs84/gravitymodel/ egm2008/first_release.html

9. O. Montenbruck and E. Gill, Satellite Orbits: Models, Methods, and Applications(2012)

10. X. Zhang and R. Piché, 2016 International Conference on Localization and GNSS (ICL-GNSS), 1-6(2016)

11. NGA GPS Ephemeris/Station/Antenna Offset Documentation, Available online: http://earthinfo.nga.mil/GandG/sathtml/gpsdoc2010 09a.html

12. P. Gérard and B. Luzum, IERS Conventions, Available online: https://www.iers.org/IERS/EN/Publications/Technic alNotes/tn36.html

13. Q. Zhang and L. Liu. Publications of the Purple Mountain Observatory, 19-27(1998) 